\title{
Ultrasound-mediated delivery of siESE complexed with microbubbles attenuates HER2+/- cell line proliferation and tumor growth in rodent models of breast cancer
}

\author{
Kang-Ho Song1, Tammy Trudeau², Adwitiya Kar², Mark A. Borden ${ }^{1 凶}$, Arthur Gutierrez-Hartmann² ${ }^{\circledR}$ \\ 1. Department of Mechanical Engineering, University of Colorado, Boulder, CO 80309, USA \\ 2. Departments of Medicine and of Biochemistry \& Molecular Genetics, University of Colorado Anschutz Medical Center, Aurora, CO 80045, USA
}

$\triangle$ Corresponding authors: Arthur Gutierrez-Hartmann, Departments of Medicine and of Biochemistry \& Molecular Genetics, University of Colorado Anschutz Medical Campus, Mail Stop 8106, Aurora, CO 80045. Phone: 303-724-3921. Email: a.gutierrez-hartmann@UCDenver.edu. Mark Borden, Department of Mechanical Engineering, University of Colorado Boulder, Mail Stop 427. Phone: 303-492-7750. Email: mark.borden@colorado.edu.

(c) Ivyspring International Publisher. This is an open access article distributed under the terms of the Creative Commons Attribution (CC BY-NC) license (https://creativecommons.org/licenses/by-nc/4.0/). See http://ivyspring.com/terms for full terms and conditions.

Received: 2018.12.10; Accepted: 2019.05.02; Published: 2019.05.13

\begin{abstract}
The highly tunable, noninvasive and spatially targeted nature of microbubble-enhanced, ultrasound-guided (MB+US) drug delivery makes it desirable for a wide variety of therapies. In breast cancer, both HER2+ and HER2- type neoplasms pose significant challenges to conventional therapeutics in greater than $40 \%$ of breast cancer patients, even with the widespread application of biologics such as trastuzumab. To address this therapeutic challenge, we examined the novel combination of tumor-injected microbubble-bound siRNA complexes and monodisperse size-isolated microbubbles (4- $\mu \mathrm{m}$ diameter) to attenuate tumor growth in vivo, as well as MB+US-facilitated shRNA and siRNA knockdown of ESE-1, an effector linked to dysregulated HER2 expression in HER2 ${ }^{+/-}$cell line propagation. We first screened six variants of siESE and shESE for efficient knockdown of ESE in breast cancer cell lines. We demonstrated efficient reduction of BT-474 (PR+, ER+, HER2+; luminal B) and MDA-MB-468 (PR-, ER-, HER2; triple-negative) clonogenicity and non-adherent growth after knockdown of ESE-1. A significant reduction in proliferative potential was seen for both cell lines using $M B+U S$ to deliver shESE and siESE. We then demonstrated significant attenuation of BT-474 xenograft tumor growth in Nod/SCID female mice using direct injection of microbubble-adsorbed siESE to the tumor and subsequent sonication. Our results suggest a positive effect on drug delivery from $M B+U S$, and highlights the feasibility of using RNAi and MB+US for breast cancer pathologies. RNAi coupled with MB+US may also be an effective theranostic approach to treat other acoustically accessible tumors, such as melanoma, thyroid, parotid and skin cancer.
\end{abstract}

Key words: ESE-1, microbubbles, ultrasound contrast agent, theranostics, gene therapy

\section{Introduction}

Cancer incidence has risen across the globe despite over half a century of investment in the development of chemotherapeutics, and the vast majority of therapeutic agents have failed to pass clinical trials (1-5). Most chemotherapeutics in current use, such as doxorubicin and paclitaxel, work by interfering with cell-cycle processes, but do so in an untargeted, transient fashion, necessitating increased doses and repeated administration, and result in significant side-effects. Treatment of some oncopathologies, such as breast cancer, have benefited from the implementation of hormone therapy and the use of targeted biologics, such as trastuzumab (anti-HER2), which target surface proteins associated with enhanced tumorigenesis in breast cancer cells. However, pathologies such as triple-negative breast cancer (TNBC) do not express genes for common breast-cancer biomarkers, and even common breast cancer pathologies may develop a resistance to antibody treatment, as evidenced by a low (30\%) response rate in $\mathrm{HER}^{+}$breast cancer patients to anti-HER2 (trastuzumab) after 5-9 months (6-8). In 
particularly aggressive oncopathologies such as HER2 positive breast cancer, circumvention of resistance to anti-HER2 therapy may involve the knockdown of positive effectors for HER2 such as epithelial-specific ETS1 (ESE-1) $(9,10)$. However, efficient delivery of siRNA and other nucleic acids has traditionally been the purview of viral agents (9), which remain limited in tissue specificity and ability to deliver sufficient quantities of therapeutic agent (11)-potentially necessitating increased doses and posing risks related to off-target effects.

Microbubble-enhanced ultrasound (MB+US) facilitates targeted drug delivery through ultrasound-mediated cavitation of lipid-encapsulated gas microspheres $(1-10 \mu \mathrm{m}$ diameter), effecting mechanofluidic disruption of tissue and cell membranes in a targeted area. Microbubbles commonly used as ultrasound contrast agents have been used as FDA-approved diagnostic agents for over a decade in contrast-enhanced sonography due to their unparalleled echogenicity under imaging ultrasound. Unlike chemically targeted treatment methods such as immunotherapy or virotherapy, $\mathrm{MB}+\mathrm{US}$ is one of few drug-delivery methods capable of spatiotemporal targeting by both chemical and physical means. The versatile nature of $\mathrm{MB}+\mathrm{US}$ drug delivery stems from several tunable parameters: the encapsulating shell may be functionalized for surface charge or conjugation with targeting ligands; the gas core and microbubble size can determine circulation persistence and magnitude of permeabilization (12-14), while ultrasonic parameters may also affect the magnitude of permeabilization. To address immunogenicity, a common issue with viral agents, several microbubble formulations incorporate a shielding layer of polyethylene-glycol (PEG), which may be further functionalized with antibodies or therapeutic payloads (15-17). A significant body of literature exists regarding enhanced treatment efficiency and spatially targeted delivery of existing chemotherapeutic agents using MB+US (18-25).

The use of gene therapy to directly suppress oncogenes or redirect immune response may be a promising alternative to aforementioned conventional chemotherapeutics, the latter highlighted by recent approval of chimeric antigen-receptor T-cell (CAR T), the first FDA-approved gene-therapy process for cancer treatment (26). However, the application of CAR $\mathrm{T}$ remains limited to few pathologies and poses significant safety challenges, such as cytokine release syndrome (CRS) - a life-threatening and systemic immune response observed in $23-80 \%$ of CAR $\mathrm{T}$ treated patients (27-30). Thus, gene therapy techniques that aim to induce oncolysis or suppress tumorigenesis remain highly relevant. Indeed,
RNAi-mediated knockdown of tumorigenic gene expression has been shown to effectively attenuate tumor growth in breast cancer cell lines resistant to conventional treatment with trastuzumab, hormone therapy and common chemotherapeutics (9).

RNAi and MB+US-mediated attenuation of tumor growth was first examined using systemically injected siEGFR+MB for squamous cell carcinoma in a 2012 study conducted by Carson et al. (31), and the approach was recently reviewed by Wang et al. in 2016 (32). In the present study, we investigate the novel combination of RNAi, MB+US drug delivery and direct tumor injection as a potential xenograft tumor-suppression method for chemotherapeuticresistant breast cancers, as well as characterize the treatment's anti-proliferative effects with two breast cancer cell lines: MDA-MB-468 (basal, triple-negative: HER2-, PR-, ER-) and BT474 (luminal B, HER2' ${ }^{+} \mathrm{PR}^{+}$, $\left.\mathrm{ER}^{+}\right)$. An in vitro sonoporation of cells in suspension was used to deliver both short-hairpin RNA (shRNA) and small-interfering RNA (siRNA) to examine RNAi efficiency and cell proliferation after MB+US. Compared to siRNA, which may be delivered to and processed in the cytoplasm, shRNA requires that its expression vector, such as pDNA, be transported to the nucleus, where intranuclear processes generate copies of the shRNA. While the translocation of pDNA introduces challenges, a potential advantage of utilizing pDNA vectors to express shRNA is the high number of replicants possible. To address nuclease-mediated degradation, we electrostatically complexed negatively charged siRNA and shRNA to positively charged (cationic) microbubbles. This siRNA+MB complex mirrors a previously investigated strategy for improving the serum stability of siRNA on nanoparticles via surface immobilization (33), and features the added benefits of tying drug delivery with microbubble destruction to improving RNA localization in the tumor. The potential combination of both imaging and drug delivery makes $\mathrm{MB}+\mathrm{US}$ an attractive theranostics approach. Finally, the majority of microbubble formulations used for MB+US are polydisperse in size, and can vary in concentration and size distribution from batch to batch (34). Based on previous studies conducted by our group regarding the effects of microbubble size $(13,14)$, we implemented monodisperse formulations of small $(2-\mu \mathrm{m})$ and medium $(4-\mu \mathrm{m})$ diameter microbubbles for in vitro cells in suspension and in vivo tumor-suppression studies, respectively.

Intratumoral delivery, particularly of immunotherapeutic agents, has drawn attention in recent years as a method to utilize a relatively small quantity of immunostimulatory agents and to achieve 
high drug concentrations within the tumor itself in a cost-effective, but more invasive manner compared to systemic delivery. $(35,36)$ Intratumoral delivery reduces systemic toxicity, and potentially harnesses lymphatic drainage to further improve drug delivery to relevant tissue-a key consideration regarding malignancy and lymphatic spread of tumoral cells.(36) In the case of MB+US delivery of interfering RNAs, intratumoral injection offers the aforementioned advantages, as well as the potential to improve RNA-propagation throughout the tumors themselves via mechanical permeabilization by the acoustically stimulated microbubbles. Indeed, previous studies have demonstrated significantly improved drug (miRNA) propagation in hepatocellular tumor tissue after MB+US, compared to $\mathrm{MBs}$ alone, reinforcing the significance of microbubble cavitation in not only intracellular delivery processes, but also tissue permeabilization and drug penetration in tumors. $(37,38)$ Although some RNA types have been identified to be stable in circulation, intratumoral injection of RNAs adsorbed to cationically charged microbubbles, as demonstrated in this study, aims to minimize the exposure of RNAs to nucleases and other degradative factors before they can take effect.

Taken together, the MB+US studies reported here underscore this approach as a novel, viable method to deliver RNAi in a highly localized manner to attenuate tumor growth. These studies are significant because they provide a treatment modality that should have fewer clinical side effects, as these agents are administrated intratumorally instead of systemically to improve the therapeutic index by achieving locally concentrated levels.

\section{Material and Methods}

\section{Size-isolated cationic microbubbles}

Cationic microbubbles were chosen owing to their ability to adsorb negatively charged nucleic acids (Fig. 1A). The cationic microbubbles were

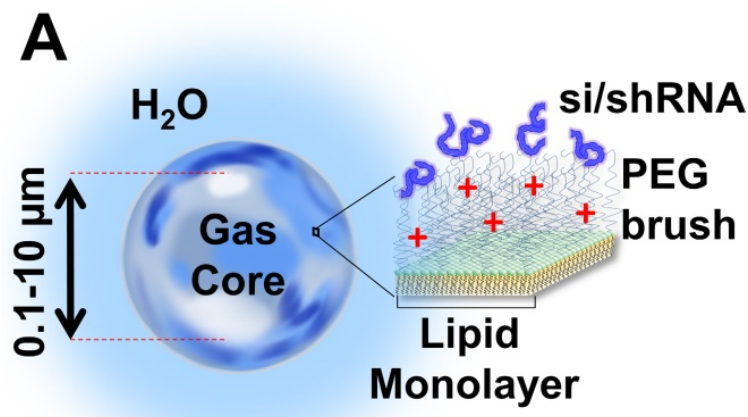

size-isolated to obtain monodisperse size-distributions to improve permeabilization (13). The cationic size-isolated microbubbles (diameters: 2 and $4 \mu \mathrm{m}$ ) used here were obtained from Advanced Microbubbles Laboratories (Boulder, CO) at concentrations between 1.8 and $2.3 \times 10^{9}$ per $\mathrm{mL}$. Microbubble size was measured with a Multisizer 3 (Beckman Coulter, Brea, CA). Cationic size-isolated microbubble formulations (referred to as 2- and 4- $\mu \mathrm{m}$ ) were measured to have median diameters of 1.7 and $4.1 \mu \mathrm{m}$ (Fig. 1B). The microbubbles were stored and handled as described in our previous study (14). The morphology and stability of cationic microbubbles when electrostatically-loaded with nucleic acids was examined in previous studies, and shown to be stable $(39,40,13)$.

\section{Cell Culture}

MDA-MB-468 is a triple-negative human breast cancer cell line. Despite our attempts, this cell line failed to grow in xenograft tumors in immune compromised mice. BT- 474 is a HER2 ${ }^{+}, \mathrm{PR}^{+}, \mathrm{ER}^{+}$ luminal B human breast cancer cell line that is capable of generating xenograft tumors in mice, and BT-474 cells were purchased from the University of Colorado Cancer Center Tissue Culture Shared Resource and short tandem repeat analysis validated stocks. MDA-MB-468 and BT-474 were cultured in DMEM/F12 supplemented with 10\% (v/v) fetal bovine serum (FBS; Gibco; Waltham, MA). For siLuc studies, BT-474 cells were first stably transfected with luciferase to generate BT-474-luc cells as previously demonstrated by our group (41). Specifically, a luciferase gene was cloned into lentiviral mammalian expression vector pCDH-CMV-MCS-puro from System Bioscience. BT474 cells were transduced with packaged virus at a ratio of $1: 3$ and cells were stably selected with puromycin at $0.5 \mathrm{ug} / \mathrm{mL}$ over a period of 1 month. Luciferase signals were tested using a luciferase assay system kit from Promega (\# E1500).

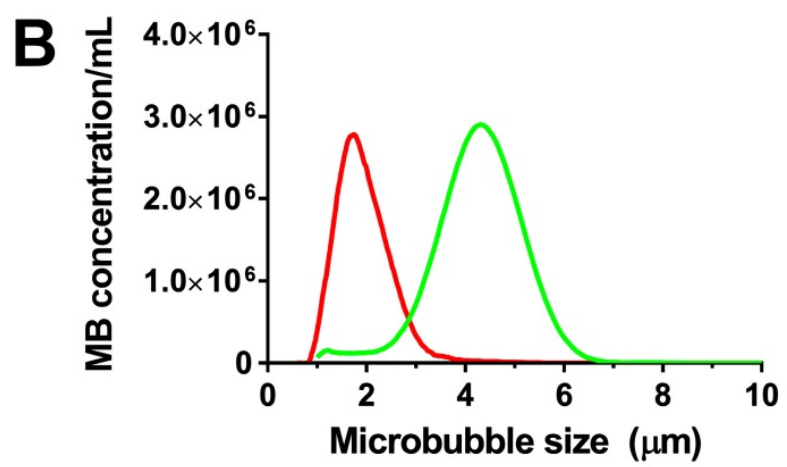

Figure 1. Cationic size-isolated microbubble drug-delivery vehicles. (A) Diagram of microbubble structure with complexed siESE/shESE. (b) Number-weighted size distribution of size-isolated microbubbles. 


\section{RNAi}

Oligonucleotides siESE-1A, B, C and D, targeting distinct domains of ESE-1, were purchased from Open Biosystems (Huntsville, AL). siESE-1_1.3 (5'-gccatgaggtactactacaaac- 3 ') targets the ETS domain and siESE-1_1.5 (5'-gcaactacttcagtgcgatgtac-3') targets the Pointed domain, and these siRNAs were custom synthesized by GE Dharmacon (Lafayette, CO). siScr is non-Targeting siRNA\#1, which was used as control (Dharmacon, Lafayette, CO). pLKO.1 shESE-1_1.5 was purchased from Open Biosystems (Huntsville, AL), and designed so that shESE-1_1.5 spanned the same target sequence as siESE-1_1.5 (5'-gcaactacttcagtgcgatgtac-3') and siMyc spanned the target sequence $5^{\prime}$-cctgagacagatcagcaacaa- $3^{\prime}$ of cMyc. c-Myc is a proto-oncogene which encodes a transcription factor associated with cellular proliferation, differentiation and apoptosis in a wide range of cells. Previous studies have shown that knockdown of c-Myc significantly reduced cell proliferation, colony formation, tumor growth and metastatsis in several cases utilizing breast-cancer cell lines such as MCF-7, MDA231, SKBR3, LY2 and MDA453.(42-44) Thus, siMyc+MB+US was chosen as a positive control to serve as an analog to siESE and to reveal the effect of degradative or cellular processes on knockdown. The shScr scrambled control was a gift from Dr. Bolin Liu (45). Also, siLuc was also purchased from Open Biosystems (Huntsville, AL). Optimal conditions for in vitro transfection and sonoporation using siRNA and shRNA were established with Lipofectamine 2000 (ThermoFisher Scientific, Waltham, MA) and MB+US, respectively.

\section{Sonoporation of cells in suspension}

Cationic microbubbles $\left(2-\mu \mathrm{m} ; 1 \times 10^{9} \mathrm{MB} / \mathrm{mL}\right)$ and siRNA $(50 \mathrm{nM})$ were mixed in $100 \mu \mathrm{L}$ of nuclease-free PBS at $4^{\circ} \mathrm{C}$ for 30 minutes to allow for adsorption of siRNA onto the microbubbles. The siRNA+MB suspension was then added to $100 \mu \mathrm{L}$ of MDA-MB-468 or BT-474 cells to achieve a total volume of $200 \mu \mathrm{L}$ with constituent concentrations of $1 \times 10^{6}$ cells $/ \mathrm{mL}, 5 \times 10^{8} \mathrm{MB} / \mathrm{mL}$ and $100 \mathrm{nM}$ siRNA. The suspension was subjected to ultrasound $(1 \mathrm{MHz}$, $2.0 \mathrm{~W} / \mathrm{cm}^{2}, 10 \%$ duty cycle) for one minute of total sonication at $37^{\circ} \mathrm{C}$ using a SoundCare Plus Clinical Ultrasound Device with a $6.5 \mathrm{~cm}^{2}$ diameter ultrasound transducer (Fig. 2). The sonoporation chamber used in these experiments was a portable version of a unit previously developed and characterized by our group using HeLa cells (13), and consisted of an acoustically-absorptive silicone barrier which held heated water, as well as an immersed cell chamber holding microbubble and cell suspension (Fig. 2). The cell chamber was constructed by cutting a
$1 \mathrm{~mL}$ Eppendorf tube (Hauppauge, NY) into a $500 \mu \mathrm{L}$ cylinder and sealing both ends with an acoustically transparent mylar film. Mixing was conducted by pausing the sonoporation at five second intervals, and timing five chamber inversions in five seconds. Sonoporated cell suspensions were plated in 6 well plates for clonogenicity and luciferase assays.

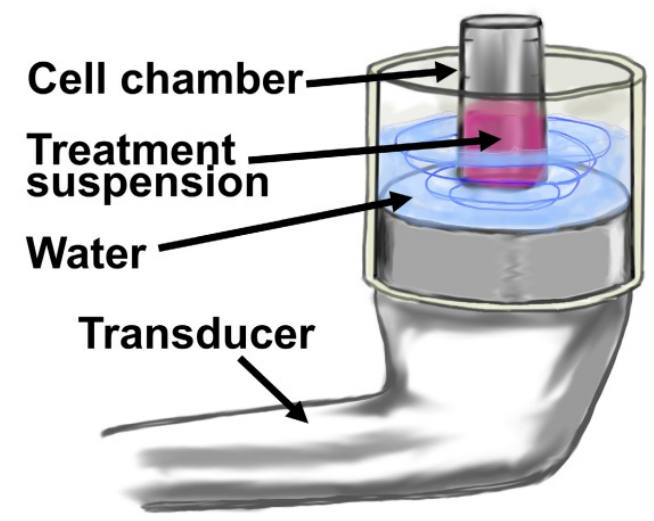

Figure 2. Diagram of in vitro $M B+U S$ configuration for transfection of cells in suspension. The treatment suspension is comprised of cell media, cells and microbubbles with adsorbed shRNA or siRNA.

\section{Clonogenicity}

Sonoporated cell suspensions were plated in 6 well tissue culture plates at 3,000 cells per well. All clonogenicity assays were incubated in a humidified tissue culture incubator at $37^{\circ} \mathrm{C}$ in $5 \% \quad \mathrm{CO}_{2}$. DMEM/F12 media was changed twice per week for 11 days total. MDA-MB-468 cells were washed the $1 \mathrm{x}$ phosphate buffered saline (PBS), fixed for $15 \mathrm{~min}$ with $4 \%$ PFA, then washed two more times with $1 x$ PBS. Colonies on tissue culture plates were stained with $0.1 \%$ crystal violet in $25 \%$ methanol for 1 hour in room temperature. Colonies were then washed twice with water and photographed with a digital camera. The acquired images were analyzed using ImageJ software (https://imagej.nih.gov/ij/). Colonies larger than $150 \mathrm{um}$ in diameter were scored as positive and counted.

\section{Soft agar assay}

Soft agar assays were performed in 6-well tissue culture plates, in assay medium that consisted of DMEM/F12 supplemented with 10\% FBS. Individual wells were covered with $1.5 \mathrm{~mL}$ of base layer $(0.6 \%)$ that was composed of $60 \%$ of agar stock ( $1 \%$ Difco TM noble agar BD \#214230 in deionized water) and 40\% of assay medium. Base layers were left to solidify for $30 \mathrm{~min}$ at room temperature. The MDA-MB-468 were grown in complete media, then harvested with trypsin and suspended in assay media. Subsequently, sonoporated cells were counted and suspended at 
10,000 cells per well. All cells suspensions were mixed with agar-containing assay media, so that the final concentration of agar was $0.3 \%$. The top layer was plated on the solidified base layers $(1.5 \mathrm{~mL}$ per well) and left to solidify for $30 \mathrm{~min}$ at room temperature. All soft agar cultures were incubated in a humidified tissue incubator at $37^{\circ} \mathrm{C}$ in $5 \% \mathrm{CO}_{2}$ for 17 days and fed with $125 \mu \mathrm{L}$ of complete media twice a week. The resulting colonies were treated with $200 \mu \mathrm{L}$ Nitroblue reagent $(1 \mathrm{mg} / \mathrm{mL}$, Amresco, \#0329) and incubated at $37^{\circ} \mathrm{C}$ overnight to develop a blue stain. Colonies were photographed with a digital camera, and the acquired images were analyzed using the ImageJ software. Colonies larger than $150 \mu \mathrm{m}$ in diameter were scored as positive and counted.

\section{Luciferase assay}

BT-474-Luc cells were resuspended at $1 \times 10^{6}$ cells/mL in DMEM/F12 media. Microbubbles were added to $2 \mathrm{~mL}$ of suspension at $1 \times 10^{8} / \mathrm{mL}$ with 100 $\mathrm{nM}$ of siLuc (Promega, Madison, WI) and sonoporated as described above. Treated cells were cultured for $48 \mathrm{hrs}$ after sonoporation, trypsinized and separated into two $40 \mu \mathrm{L}$ wells. Luciferase $(40 \mu \mathrm{L})$ was added to each well and luminescence was measured using a Synergy HTX plate reader (Biotek, Winooski, VT).

\section{Immunocytochemistry}

MDA-MB-468 were harvested in $0.05 \%$ trypsin with $0.5 \mathrm{mM}$ EDTA and 3,000 cells were plated in 8 -well chamber slides. Cells were washed with $1 x$ PBS, fixed for $15 \mathrm{~min}$ in 4\% PFA, then washed two more times with $1 x$ PBS. Cells were permeabilized with $0.1 \%$ Triton X-100 in PBS, and then blocked for 30 $\mathrm{min}$ in PBS $/ 0.2 \%$ BSA. The slide was incubated overnight with anti-ESE-1 (mAb405 generated in the AGH lab) in PBS/0.2\% BSA, washed 3 times with PBS $/ 0.2 \%$ BSA, and then incubated with secondary antibody (Biotinylated goat anti-Mouse IgG Vector Laboratories) in PBS $/ 0.2 \%$ BSA. Thereafter the slides were washed in PBS/0.2\%BSA, and then Vectastain $A B C$ is added for 30 mins. Cells are then washed 3 times with PBS $/ 0.2 \%$ and then the treatment of $\mathrm{DAB}+$ peroxidase substrate (DAKO, \# K3468) per manufacturer's instructions. Slides were washed in water and then nuclei were counterstained with Mayer's hematoxylin (diluted 1:10) for 30s. Thereafter, the slide was rinsed in water, dehydrated in graded ethanol and then mounted using Permount (Fisher \# SP15-100).

\section{Animal procedures}

Animal procedures were approved by the University of Colorado Denver IACUC committee (Protocol \# 63814(12)1E). Female NOD.CB17-
Prkdcscid/NCrHsd (NOD.SCID) mice (5-6 weeks) were obtained from NCI-Frederick. After one week of acclimation the mice were subcutaneously implanted with silastic pellets containing cellulose $/ 17 \beta$-estradiol mixture ( $2 \mathrm{mg} /$ pellet). After two weeks, $2 \times 10^{6}$ cells BT474-Luc cells were resuspended with $50 \mu \mathrm{L}$ of Matrigel (BD Biosciences \#356230) and were injected bilaterally onto mammary fat pads. A total of 6 separate rounds of injections (6 distinct biological replicates) were performed. Each round included from 4 to 15 mice, with the following groups represented: siScr (negative control), siESE-1.3, siMyc (positive control), siESE-1.3 -MB +US, and siMyc -MB +US, for a total of 50 mice and 59 tumors.

Once tumors became palpable, initial tumor volume was calculated using caliper measurements. Around 4 weeks post-cell-line/Matrigel injections, when tumors achieved $\sim 100 \mathrm{~mm}^{3}$, they were injected with a mixture of either $10 \mu \mathrm{g}$ siControl, siESE-1, or sicMyc in $25 \mu \mathrm{L}$, plus $25 \mu \mathrm{L}$ microbubble, for a total volume of $50 \mu \mathrm{L}$. The mixture was then injected directly into the center of the tumor. Ultrasonic imaging was considered to assess the proliferation of microbubbles throughout the tumor, as has been shown in prior studies (46). However, this was deemed unnecessary for intratumoral delivery because a direct visual observation could be made on the propagation and distribution of microbubbles within the tumor mass, owing to their highly refractive, white color. Tumors were then subjected to ultrasound ( $1 \mathrm{MHz}, 2.0 \mathrm{~W} / \mathrm{cm}^{2}, 10 \%$ duty cycle) for 2 minutes using the SoundCare Plus Clinical Ultrasound Device with the 1- $\mathrm{cm}^{2}$ diameter ultrasound transducer. Tumor-targeted MB+siESE injections were repeated after $24 \mathrm{hrs}$, and $48 \mathrm{hrs}$ from the initial injection (for a total of 3 injections). After the third injection, tumors were measured with calipers for two weeks. Successful injection of complexed MB+siRNA was observed as a conspicuous lightening of the tumor under the shaved skin of the host mice due to distribution of the microbubble mixture. No significant weight change or behavioral anomalies were observed in MB+US-treated animals.

Tumor sizes were measured as tumors became palpable, and tumor growth in response to control and siESE-treated conditions was evaluated for a range of initial tumor volumes $\left(33.5-1230.9 \mathrm{~mm}^{3}\right)$ (See Supplemental Data). Initial tumor volume was determined after tumors grew to palpable volumes, with $150 \mathrm{~mm}^{3}$ targeted as the initial tumor volume, and an actual measured median of $131.9 \mathrm{~mm}^{3}$ and a mean of $226.7 \mathrm{~mm}^{3}$. Final tumor volume was measured two weeks from this initial measurement and normalized to the initial measurement (See 
Supplemental Data). Growth was represented as the final tumor volume divided by initial tumor volume.

\section{Data analysis}

Normalized clonogenicity and proliferation of cells on soft-agar were represented as the final number of colonies divided by the original number of colonies. Normalized tumor growth at two weeks was obtained by dividing the two-week tumor volume by initial tumor volume. Statistical analysis was conducted in GraphPad Prism (La Jolla, CA), and statistical significance between samples representing two conditions were analyzed using a Mann-Whitney test, with $p<0.05$ interpreted as a significantly different comparison.

\section{Results}

\section{ESE-1 knockdown is achieved in HER2+, ER+, luminal $B$ human breast cancer cell lines using siESE 1 and $M B+U S$}

ESE-1 knockdown was achieved in two breast cancer cell lines, MDA-MB-468 and BT-474, which represented triple-negative (ER-, PR-, HER2-; basal) and conventional $\left(\mathrm{ER}^{+}, \mathrm{PR}^{+}, \mathrm{HER} 2^{+}\right.$; luminal) breast cancer cell types, respectively. We first targeted for ESE-1 sequences that would achieve optimal ESE-1 knockdown in MDA-MB-468 cells, by transfecting with six distinct sequences of siESE $(1 \mathrm{~A}, 1 \mathrm{~B}, 1 \mathrm{C}, 1 \mathrm{D}$, 1.3 and 1.5) using Lipofectamine-2000. Immunoblot of MDA-MB-468 cells transfected with three siESE-1 variants $(-1 C, 1.3$ and 1.5) using Lipofectamine-2000 demonstrated significantly reduced anti-ESE staining compared to the negative control, si-scramble (siScr), and siESE-1A, $-1 \mathrm{~B}$ and $-1 \mathrm{D}$ (Fig. 3A). Immunblotting for tubulin was performed to control for sample loading. siESE-1_1.3 qualitatively demonstrated an increase in ESE knockdown over siESE-1_1.5 (Fig. $3 \mathrm{~A})$, and based upon these results, si/shESE-1_1.3 was selected for subsequent MB+US-attenuated colony formation and tumor growth. Similar to lipofectamine+siESE treated conditions, sonoporation-mediated MB+US+siESE-1_1.3 effected significantly reduced anti-ESE levels in BT-474 cell lysates (Fig. 3B), and microbubbles were required to enhance sonoporation (Fig. 3C). To determine whether plasmids expressing shRNA could also be sonoporated using microbubbles, MDA-MB-468 cells treated with short-hairpin RNA targeting ESE (shESE-1_1.5), and MB+US were also observed to express significantly less ESE-1 as determined by immunocytochemistry than cells treated with MB+US and shScr or samples treated with ultrasound alone (Fig. 3D and 3E).
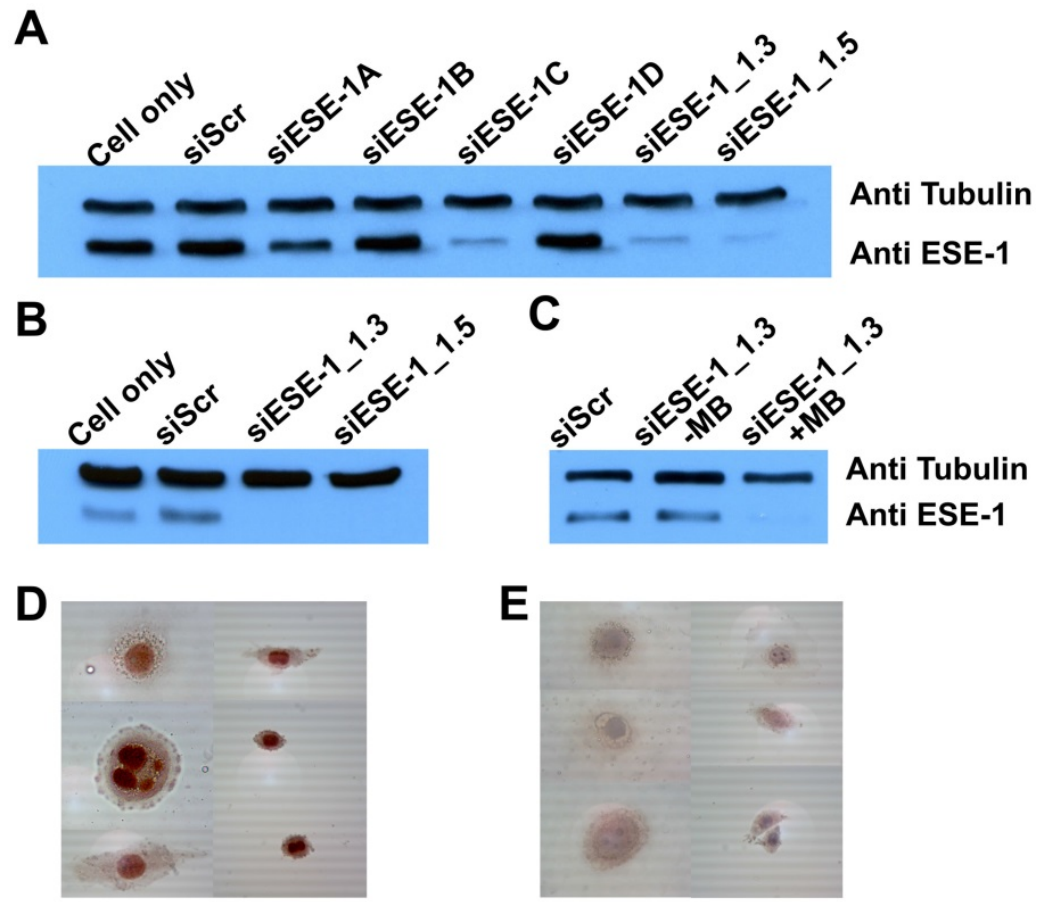

Figure 3. Comparative ESE-1 expression and MB+US knockdown using siESE-1 and shESE-1. (A) Western blot analysis of ESE-1 knockdown via lipofectamine siRNA transfection. MDA-MB-468 triple-negative breast cancer cells were transfected with Lipofectamine and siScr, six variants of siESE for optimization. (B) Western blot analysis of ESE-1 knockdown via MB+US sonoporation. MB+US was used to transfect MD-MB-468 cells with siESE-1. (C) Assessing requirement of $M B$ for the sonoporation of siESE-1_1.3. US+/-MB was also used to transfect BT-474 luminal $B$ breast cancer cells with siESE-1_1.3. (D and E) Immunocytochemistry analysis of ESE-1 knockdown via sonoporation in MDA-MB-468 cells. A plasmid construct encoding shESE-1 1.5 was delivered using MB+US targeting scramble (Scr) (D) or ESE-1 (shESE-1_1.5) (D). ICC was conducted with anti-ESE-1 mAb405 and developed with secondary Ab. ESE-1 levels show as a red stain. 
A

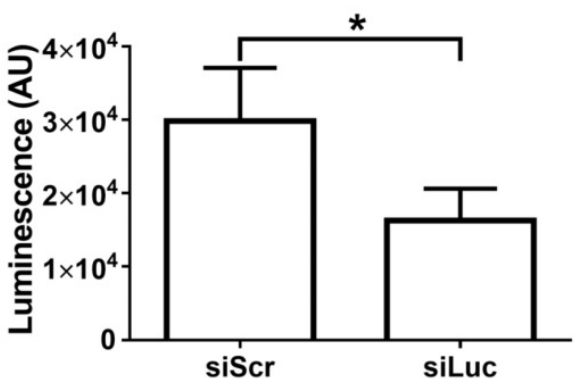

B

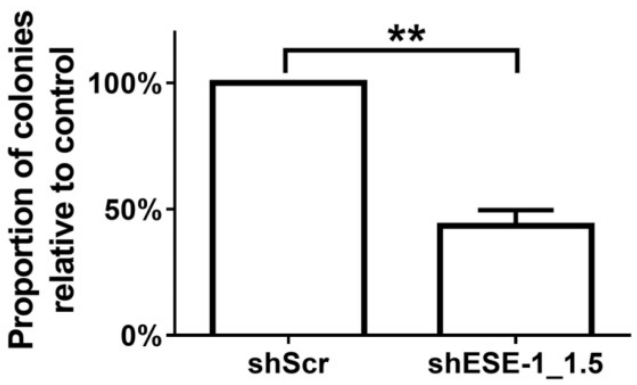

C

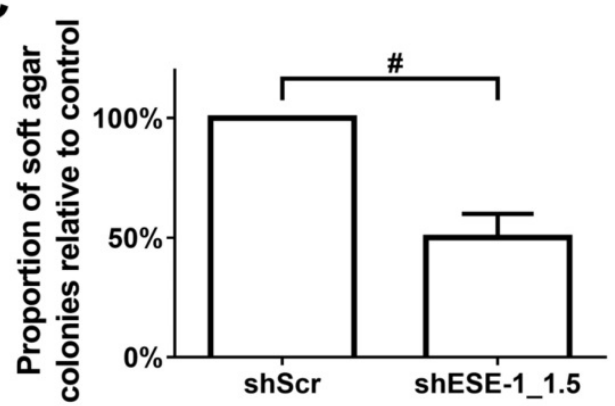

Figure 4. Quantification MB/US-mediated siRNA knockdown of ESE-1 and its effects on the transformation features of BT-474-Luc and MDA-MB-468 breast cancer cell lines. (A) Luminescence reduction was evaluated $48 \mathrm{hrs}$ after $\mathrm{MB}+\mathrm{US}$-sonoporated siLuc delivery to suspended, BT-474-Luc cells stably expressing luciferase. (B) MDA-MB-468 colony formation on tissue culture plates was evaluated after MB+US-facilitated delivery of shESE-1_1.5. (C) Soft-agar colony numbers were evaluated 11 days after MB+US-facilitated delivery of shESE-1 1.5 to MDA-MB-468 cells in suspension $\left(^{*}, \mathrm{p}<0.05\right.$; **, $\mathrm{p}<0.0001 ; \#, \mathrm{p}<0.001)$.
MB+US-facilitated ESE- 1 knockdown in breast cancer cell lines attenuates colony formation

Quantitative evaluation of MB+US-mediated transfection efficiency was initially evaluated with siLuc in BT-474-luc cells (Fig. 4A). A 45.5\% reduction of luminescence between control (siScr) and siLuc-treated cells was observed in cells $48 \mathrm{hrs}$ after $\mathrm{MB}+\mathrm{US}$, indicating intracellular delivery of siRNA via MB+US. We next evaluated adhesion and adhesion-independent cell growth by examining clonogenicity and soft-agar colony formation, respectively, in MDA-MB-468 cells 17 days after MB+US-mediated transfection with siScr and shESE-1_1.3. Compared to control (siScr-transfected cells), MB+US-facilitated delivery of shESE-1_1.3 to MDA-MB- 468 cells resulted in a $56.7 \pm 6.2 \%$ decrease in colony formation in adherent conditions (clonogenicity assay, Fig. 4B) and a $50.0 \pm 10.1 \%$ decrease of nonadhesive colony formation in soft agar (Fig. 4C), indicating successful shESE-1_1.3 delivery, ESE-1 knockdown, and reduction in transformation properties.

\section{MB+US-facilitated attenuation of tumor growth after direct injection of MB and siESE}

A preliminary comparison of implanted MDA-MB-468-luc and BT-474-luc tumorigenicity in NOD SCID female mice indicated significantly increased tumor formation with BT-474-luc cells (data not shown). Therefore, BT-474-luc was selected to be the seeded cells for in vivo tumorigenesis and tumor growth studies. c-Myc, a "master regulator" oncogene whose knockdown is strongly linked with reduced tumorigenesis in several pathologies (33-39), was targeted in positive controls in conditions using $\mathrm{MB}+\mathrm{US}$ and siMyc. siMyc+MB+US conditions demonstrated a $41.7 \%$ reduction $(p<0.10)$ in tumor growth compared to siScr-treated tumors (Fig. 6). Injection of siMyc alone, without microbubbles, resulted in a $19.3 \%$ reduction $(p>0.50)$ in tumor volume size compared to siScr conditions.
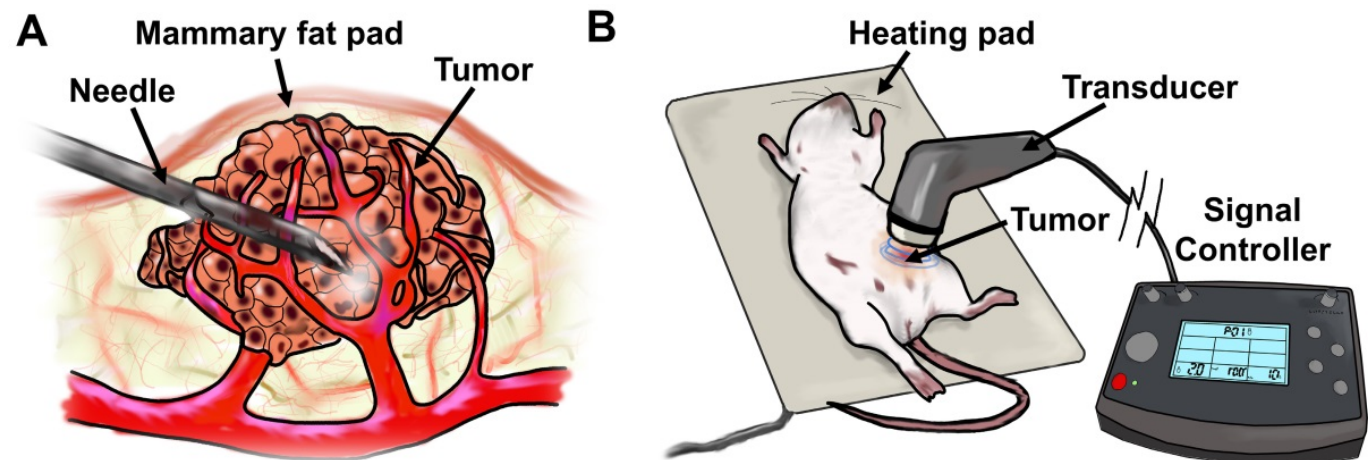

Figure 5. Diagram of tumor-targeted MB+US process. a) direct-injection of complexed siESE-1_1.3 and cationic size-isolated microbubbles, and (b) ultrasound application to $\mathrm{MB}+$ siRNA-injected tumors. 
Application of ultrasound and MB+siESE-1_1.3 resulted in significantly smaller tumor growth (3.8 \pm 1.5 -fold growth) compared to negative control tumors treated with siScr (5.9 \pm 3.3 -fold growth), effecting a $35.6 \%$ reduction in tumor size $(\mathrm{p}<0.05$; Fig. 6B). Ultrasound application after siESE-1_1.3 injection without microbubbles resulted in an $18.6 \%$ reduction $(p>0.3)$ in tumor size compared to siScr

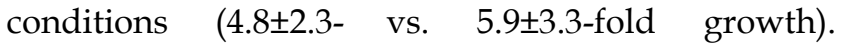
Comparison of tumor volume after treatment with positive control conditions (MB+US+siMyc; 3.5 \pm 2.5 -fold growth) and tumor volume after treatment with $\mathrm{MB}+\mathrm{US}+\mathrm{siESE}$ (3.8 \pm 1.5 -fold growth) showed no significant difference $(\mathrm{p}>0.7)$.

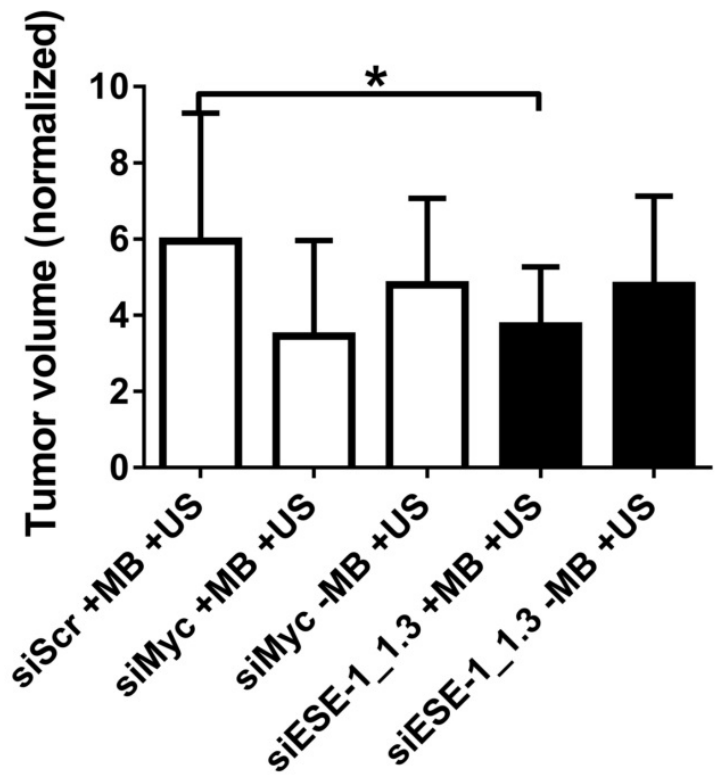

Figure 6. Tumor growth attenuation with MB+US-mediated delivery of siESE-1_1.3 directly into the tumor. BT-474-Luc xenograft tumor growth was evaluated in NOD/SCID female mice after siRNA direct injection into the tumors followed by MB+US delivery into tumor cells (*, $p<0.05$ ). BT-474-Luc cells seeded in Matrigel plugs were injected into mammary fat pads, and tumor growth was measured as tumors became palpable. Initial tumor volume was recorded, and it ranged from 33.5-1230.9 $\mathrm{mm}^{3}$ (with initial tumor size having a median size of $131.9 \mathrm{~mm}^{3}$ and a mean size of $226.7 \mathrm{~mm}^{3}$ ). Final tumor volume was recorded two weeks after initial tumor volume and the data are presented as fold-increase of final volume over initial volume. The data in this figure is derived from six distinct injection studies (ie, six biological replicates, representing 50 mice and 59 tumors), and is represented over time (A) and after two weeks (B).

\section{Discussion}

An initial goal of this study was to utilize MB+US to achieve targeted delivery of si/shESE and efficient knockdown of ESE-1 in two distinct breast cancer cell lines, and to characterize the anti-proliferative efficiency of MB+US-facilitated delivery of siRNA and shRNA in vitro and siRNA in xenograft tumors. Colony-forming behavior in cancerous cells not adhered to a surface is a well-documented characteristic of tumorigenesis
$(54,55)$. Our results indicated that MB+US-facilitated siESE knockdown of ESE-1 resulted in decreased colony formation in both adherent (plated on etched polystyrene surface) as well as non-adherent (soft agar surface) cells (Fig. 3 and 4). Specifically, successful knockdown of ESE-1 and the resulting anti-proliferative effect was achieved in vitro with two cell types (MDA-MB-468 and BT474), two transfection methods (liposomal and MB+US delivery), and siESE as well as shESE. The reduction in proliferative behavior after si/shRNA+MB+US treatment for these two disparate cell lines was particularly interesting: MDA-MB-468 is a basal cell type, negative for estrogen receptor (ER), progesterone receptor (PR), and human epidermal growth factor receptor 2 (HER2). In contrast, BT-474 is a luminal-type cell line and is generally positive for ER, PR and HER2. Although ESE-1 expression has previously been shown to correlate with HER2 expression in human breast cancers (56-59), it remains possible that ESE-1 knockdown not only decreases HER2 and pHER2 levels (9), but may also affect other signaling pathways to mediate the anti-tumorigenic effects of reduced ESE-1 levels. Additionally, the ability of MB+US to efficiently deliver shESE-1_1.5 and achieve an anti-proliferative effect in vitro was of note (Fig. 4), due to the larger size of plasmid vectors encoding short-hairpin RNAs (> $4 \mathrm{MDa}$ ) compared to siRNA (< $8 \mathrm{kDa}$ ), as well as the need to localize plasmid vectors encoding shRNA in the nucleus to effect transfection. Compared to electroporation which permeabilizes both the nuclear and cell membrane, it is likely that MB+US effects transient permeabilization of the cell membrane alone $(60,61)$. While si/shRNA integrity was not characterized after adsorption to the microbubble, knockdown efficacy in clonogenicity and soft-agar assays was interpreted as evidence that nucleic acids were functionally preserved during the experimental procedures. It is also important to note that the shRNA mentioned in our study was expressed from pDNA. In a previous study examining BT-474 tumor transduction using lentivirus-packaged shESE-1_1.3, our group saw an $86 \%$ reduction in tumor growth compared to shSCR-treated controls $(9,41)$. Our current study demonstrates a $40 \%$ reduction with siESE delivered from microbubbles. We attribute this discrepancy to the potential transduction efficiency of viruses, as well as the use of siESE instead of shESE. Specifically, siESE was selected due to the higher molar concentrations of loading possible on the surface of the microbubble compared to the much larger pDNA expressing shRNA. The payload limitations specific to pDNA/shESE could be addressed in future studies, for example by using layer-by-layer loading of shESE 
on the microbubble shell (40), polyplexes (46), lipoplexes (62), or other strategies.

Several elegant studies have shown that MB+US enhances chemotherapeutic delivery to tumors in regards to systemically circulating therapeutics $(23,25,63-67)$, and that the mechanism for enhanced delivery is similar to the tissue-disruption mediated drug extravasation found in blood-brain-barrier opening $(14,68)$. This study examined direct injection of complexed MB payload to the interstitium of the xenograft BT-474-Luc tumor, which when coupled with MB+US-mediated destruction of the microbubbles, was expected to similarly permeabilize tumor tissue and improve drug propagation. Compared to systemically-injected microbubbles, which have been shown to experience clearance due to immune factors or sequestering in the lung, liver, kidneys and spleen $(69,70)$, a distinct advantage of intratumorally-delivered microbubbles is the enhanced localization of drug in the tumor body itself, and possible reduction in off-target effects compared to systemic injection. It is important to note that only a handful of MB+US studies have targeted oncogene expression (71-73), and such studies have utilized free-floating viral or pDNA payloads which have been systemically injected with microbubbles. The accelerated degradation of RNA compared to DNA in the bloodstream partially motivated our approach regarding direct tumor injection and electrostatic adsorption of RNA to MBs. Compared to shRNA-mediated knockdown, siRNA-mediated suppression was believed to be a more reliable effector of knockdown in conjunction with MB+US, due to higher molar concentration loaded onto the microbubbles, albeit at a shorter duration. We therefore selected siRNA for the tunable knockdown of ESE-1 in our tumor-growth attenuation study, and implemented serial treatments.

In this study, we conduct intratumoral injection of MBs, which seems well-suited for the delivery of RNAs in breast cancers; this delivery scheme offers a method of reducing RNA exposure to a multitude of nucleases compared to systemic delivery, and the impact of its invasiveness is perhaps lessened due to the nature and location of breast tumors. Additionally, the successful use of siESE in these studies is a novel application of ESE-1-mediated oncotherapy in triple-negative breast cancers, and the reduction in tumor growth achieved with $\mathrm{MB}+\mathrm{US}$ and siESE demonstrates a viable alternative to viral delivery. Opportunities certainly exist for delivering other non-viral payloads: biologics, such as trastuzumab, may demonstrate improved efficiency when localized via intratumoral injection and dispersed using MB+FUS.
Finally, the safety of MB+US-mediated delivery has been a topic of some study, particularly in the treatment of volumes sensitive to insult such as brain tissue (74-76). While tissue damage was not a concern in this study, a consideration in the treatment of tumor pathologies is ultrasound propagation around the targeted site, resulting in $\mathrm{MB}$ destruction, subsequent release of payload and tissue permeabilization. We propose the following methods to potentially mitigate off-target effects with MB+US: 1) Injecting the therapeutic agent and $M B$ directly into the treatment site or tumor resection cavity, 2) complexing the therapeutic agent with microbubbles using electrostatic or chemical interactions to enable exclusive release of payload upon microbubble destruction or dissolution, 3) initiating ultrasound stimulation immediately after direct injection of the tumor, and 4) utilizing focused ultrasound or appropriately dimensioned ultrasound beamforms to minimize stimulation of non-tumor tissue.

\section{Conclusion}

MB+US-mediated sonoporation delivery of siESE adsorbed to cationic microbubbles was demonstrated to be an effective means of significantly attenuating tumor growth in BT-474-Luc xenograft tumors after direct injection of siRNA-MB complex. Additionally, ESE-1 expression, as well as 2D colony and $3 \mathrm{D}$ soft agar colony formation was shown to be significantly reduced in both $\mathrm{BT}-474\left(\mathrm{ER}^{+}, \mathrm{PR}^{+}\right.$, HER2 $^{+}$) and MDA-MB-468 (ER-, PR-, HER2-) after $\mathrm{MB}+\mathrm{US}$-delivery of shESE or siESE. We conclude that future oncogene-suppressing $\mathrm{MB}+\mathrm{US}$ therapy using siRNA may be a promising means of treating challenging breast-cancer pathologies, as well as sub-epidermal tumors such as melanoma, thyroid, parotid and skin cancer.

\section{Supplementary Material}

Supplementary data.

http://www.ntno.org/v03p0212s1.pdf

\section{Acknowledgements}

Funding for this study was provided by a grant from the Colorado Clinical and Translational Sciences Institute to AGH and MAB, as well as NIH R01 CA141201 to AGH and NIH R01 CA195051 to MAB.

\section{Competing Interests}

The authors have declared that no competing interest exists.

\section{References}

1. Fojo T, Amiri-Kordestani L, Bates SE. Potential Pitfalls of Crossover and Thoughts on Iniparib in Triple-Negative Breast Cancer. JNCI J Natl Cancer Inst. 2011 Dec 7;103(23):1738-40. 
2. Amiri-Kordestani L, Fojo T. Why Do Phase III Clinical Trials in Oncology Fail so Often? JNCI J Natl Cancer Inst. 2012 Apr 18;104(8):568-9.

3. Stensland KD, McBride RB, Latif A, Wisnivesky J, Hendricks R, Roper N, et al. Adult Cancer Clinical Trials That Fail to Complete: An Epidemic? JNCI J Natl Cancer Inst [Internet]. 2014 Sep [cited 2018 Jul 24];106(9). Available from: https://academic.oup.com/jnci/article-lookup/doi/10.1093/jnci/dju229

4. Amiri-Kordestani L, Basseville A, Kurdziel K, Fojo AT, Bates SE. Targeting MDR in breast and lung cancer: Discriminating its potential importance from the failure of drug resistance reversal studies. Drug Resist Updat. 2012 Feb;15(1-2):50-61.

5. Maeda H, Khatami M. Analyses of repeated failures in cancer therapy for solid tumors: poor tumor-selective drug delivery, low therapeutic efficacy and unsustainable costs. Clin Transl Med [Internet]. 2018 Dec [cited 2018 Jul 24];7(1). Available from: https://clintransmed.springeropen.com/ articles/10.1186/s40169-018-0185-6

6. Gijsen M, King P, Perera T, Parker PJ, Harris AL, Larijani B, et al. HER2 Phosphorylation Is Maintained by a PKB Negative Feedback Loop in Response to Anti-HER2 Herceptin in Breast Cancer. Kemp C, editor. PLoS Biol. 2010 Dec 21;8(12):e1000563.

7. Madrid-Paredes A, Cañadas-Garre M, Sánchez-Pozo A, Calleja-Hernández MÁ. Non-HER2 signaling pathways activated in resistance to anti-HER2 therapy in breast cancer. Breast Cancer Res Treat. 2015 Oct;153(3):493-505.

8. Luque-Cabal M, García-Teijido P, Fernández-Pérez Y, Sánchez-Lorenzo L, Palacio-Vázquez I. Mechanisms behind the Resistance to Trastuzumab in HER2-Amplified Breast Cancer and Strategies to Overcome It. Clin Med Insights Oncol. 2016 Jan;10s1:CMO.S34537.

9. Adwitiya K, Liu B, Gutierrez-Hartmann A. ESE-1 Knockdown Attenuates Growth in Trastuzumab-resistant HER2+ Breast Cancer Cells. Anticancer Res [Internet]. 2017 Dec 3 [cited 2018 Jul 25];37(12). Available from: http://ar.iiarjournals.org/content/37/12/6583.abstract

10. Prescott JD, Poczobutt JM, Tentler JJ, Walker DM, Gutierrez-Hartmann A. Mapping of ESE-1 subdomains required to initiate mammary epithelial cell transformation via a cytoplasmic mechanism. Mol Cancer. 2011;10(1):103.

11. Pardridge WM, Boado RJ. Reengineering biopharmaceuticals for targeted delivery across the blood-brain barrier. Methods Enzymol. 2012;503:269-92.

12. Sirsi S, Feshitan J, Kwan J, Homma S, Borden M. Effect of microbubble size on fundamental mode high frequency ultrasound imaging in mice. Ultrasound Med Biol. 2010;36(6):935-948.

13. Song K-H, Fan AC, Brlansky JT, Trudeau T, Gutierrez-Hartmann A, Calvisi ML, et al. High Efficiency Molecular Delivery with Sequential Low-Energy Sonoporation Bursts. Theranostics. 2015;5(12):1419-27.

14. Song K-H, Fan AC, Hinkle JJ, Newman J, Borden MA, Harvey BK. Microbubble gas volume: A unifying dose parameter in blood-brain barrier opening by focused ultrasound. Theranostics. 2017;7(1):144-52.

15. Chen CC, Borden MA. Ligand Conjugation to Bimodal Poly(ethylene glycol) Brush Layers on Microbubbles. Langmuir. 2010 Aug 17;26(16):13183-94.

16. Chen CC, Borden MA. The Role of Poly(ethylene glycol) Brush Architecture in Complement Activation on Targeted Microbubble Surfaces. Biomaterials. 2011 Sep;32(27):6579-87.

17. Wu S-Y, Chen CC, Tung Y-S, Olumolade OO, Konofagou EE. Effects of the microbubble shell physicochemical properties on ultrasound-mediated drug delivery to the brain. J Control Release Off J Control Release Soc. 2015 Aug 28;212:30-40.

18. Wu J, Pepe J, Rincón M. Sonoporation, anti-cancer drug and antibody delivery using ultrasound. Ultrasonics. 2006;44:e21-e25.

19. Iwanaga K, Tominaga K, Yamamoto K, Habu M, Maeda H, Akifusa S, et al. Local delivery system of cytotoxic agents to tumors by focused sonoporation. Cancer Gene Ther. 2007;14(4):354-363.

20. Treat LH, McDannold N, Zhang Y, Vykhodtseva N, Hynynen K. Improved Anti-Tumor Effect of Liposomal Doxorubicin After Targeted Blood-Brain Barrier Disruption by MRI-Guided Focused Ultrasound in Rat Glioma. Ultrasound Med Biol. 2012 Oct:38(10):1716-25.

21. Treat LH, McDannold N, Vykhodtseva N, Zhang Y, Tam K, Hynynen K. Targeted delivery of doxorubicin to the rat brain at therapeutic levels using MRI-guided focused ultrasound. Int J Cancer. 2007 Aug 15;121(4):901-7.

22. Escoffre J, Mannaris C, Geers B, Novell A, Lentacker I, Averkiou M, et al. Doxorubicin liposome-loaded microbubbles for contrast imaging and ultrasound-triggered drug delivery. IEEE Trans Ultrason Ferroelectr Freq Control [Internet]. 2013 Jan [cited 2015 Nov 16];60(1). Available from: http://ieeexplore.ieee.org/lpdocs/epic03/wrapper.htm?arnumber=6396488

23. Kotopoulis S, Dimcevski G, Gilja OH, Hoem D, Postema M. Treatment of human pancreatic cancer using combined ultrasound, microbubbles, and gemcitabine: a clinical case study. Med Phys. 2013 Jul;40(7):072902.

24. Kovacs Z, Werner B, Rassi A, Sass JO, Martin-Fiori E, Bernasconi M. Prolonged survival upon ultrasound-enhanced doxorubicin delivery in two syngenic glioblastoma mouse models. J Controlled Release. 2014 Aug:187:74-82.

25. Kotopoulis S, Delalande A, Popa M, Mamaeva V, Dimcevski G, Gilja OH, et al. Sonoporation-enhanced chemotherapy significantly reduces primary tumour burden in an orthotopic pancreatic cancer xenograft. Mol Imaging Biol MIB Off Publ Acad Mol Imaging. 2014 Feb;16(1):53-62.

26. Almåsbak H, Aarvak T, Vemuri MC. CAR T Cell Therapy: A Game Changer in Cancer Treatment. J Immunol Res. 2016;2016:1-10.

27. Brentjens RJ, Davila ML, Riviere I, Park J, Wang X, Cowell LG, et al. CD19-Targeted T Cells Rapidly Induce Molecular Remissions in Adults with
Chemotherapy-Refractory Acute Lymphoblastic Leukemia. Sci Transl Med. 2013 Mar 20;5(177):177ra38-177ra38

28. Davila ML, Riviere I, Wang X, Bartido S, Park J, Curran K, et al. Efficacy and Toxicity Management of 19-28z CAR T Cell Therapy in B Cell Acute Lymphoblastic Leukemia. Sci Transl Med. 2014 Feb 19;6(224):224ra25-224ra25.

29. D'Aloia MM, Zizzari IG, Sacchetti B, Pierelli L, Alimandi M. CAR-T cells: the long and winding road to solid tumors. Cell Death Dis [Internet]. 2018 Mar [cited 2018 Jul 26];9(3). Available from: http://www.nature.com/ articles/s41419-018-0278-6

30. Althoff E, Phillips F. Kymriah ${ }^{\circledR}$ (tisagenlecleucel), first-in-class CAR-T therapy from Novartis, receives second FDA approval to treat appropriate $\mathrm{r} / \mathrm{r}$ patients with large B-cell lymphoma [Internet]. 2018 May. Available from: https://www.novartis.com/news/media-releases/kymriahr-tisagenlecleucel -first-class-car-t-therapy-from-novartis-receives-second-fda-approval-treat-ap propriate-rr-patients-large-b-cell-lymphoma

31. Carson AR, McTiernan CF, Lavery L, Grata M, Leng X, Wang J, et al. Ultrasound-Targeted Microbubble Destruction to Deliver siRNA Cancer Therapy. Cancer Res. 2012 Dec 1;72(23):6191-9.

32. Wang J, Mi P, Lin G, Wáng YXJ, Liu G, Chen X. Imaging-guided delivery of RNAi for anticancer treatment. Adv Drug Deliv Rev. 2016 Sep 1;104:44-60.

33. Barnaby SN, Lee A, Mirkin CA. Probing the inherent stability of siRNA immobilized on nanoparticle constructs. Proc Natl Acad Sci. 2014 Jul 8;111(27):9739-44.

34. Goertz DE, de Jong N, van der Steen AFW. Attenuation and Size Distribution Measurements of Definity ${ }^{\mathrm{TM}}$ and Manipulated Definity ${ }^{\mathrm{TM}}$ Populations. Ultrasound Med Biol. 2007 Sep;33(9):1376-88.

35. Marabelle A, Tselikas L, de Baere T, Houot R. Intratumoral immunotherapy: using the tumor as the remedy. Ann Oncol.2017 Dec 1;28(suppl_12):xii33-43.

36. Aznar MA, Tinari N, Rullán AJ, Sánchez-Paulete AR, Rodriguez-Ruiz ME, Melero I. Intratumoral Delivery of Immunotherapy-Act Locally, Think Globally. J Immunol. 2017 Jan 1;198(1):31-9.

37. Wang T-Y, Choe JW, Pu K, Devulapally R, Bachawal S, Machtaler S, et al. Ultrasound-guided delivery of microRNA loaded nanoparticles into cancer. J Controlled Release. 2015 Apr;203:99-108.

38. Mullick Chowdhury S, Wang T-Y, Bachawal S, Devulapally R, Choe JW, Abou Elkacem L, et al. Ultrasound-guided therapeutic modulation of hepatocellular carcinoma using complementary microRNAs. J Controlled Release. 2016 Sep;238:272-80.

39. Christiansen JP, French BA, Klibanov AL, Kaul S, Lindner JR. Targeted tissue transfection with ultrasound destruction of plasmid-bearing cationic microbubbles. Ultrasound Med Biol. 2003 Dec;29(12):1759-67.

40. Borden MA, Caskey CF, Little E, Gillies RJ, Ferrara KW. DNA and Polylysine Adsorption and Multilayer Construction onto Cationic Lipid-Coated Microbubbles. Langmuir. 2007 Aug;23(18):9401-8.

41. Kar A, Gutierrez-Hartmann A. ESE-1/ELF3 mRNA expression associates with poor survival outcomes in HER2+ breast cancer patients and is critical for tumorigenesis in HER2+ breast cancer cells. Oncotarget [Internet]. 2017 Sep 19 [cited 2018 Aug 2];8(41). Available from: http://www.oncotarget.com/fulltext/18710

42. Wang Y, Liu S, Zhang G, Zhou C, Zhu H, Zhou X, et al. Knockdown of c-Myc expression by RNAi inhibits MCF-7 breast tumor cells growth in vitro and in vivo. Breast Cancer Res BCR. 2005;7(2):R220-228.

43. Wolfer A, Wittner BS, Irimia D, Flavin RJ, Lupien M, Gunawardane RN, et al. MYC regulation of a "poor-prognosis" metastatic cancer cell state. Proc Natl Acad Sci. 2010 Feb 23:107(8):3698-703.

44. Zhang X, Farrell AS, Daniel CJ, Arnold H, Scanlan C, Laraway BJ, et al. Mechanistic insight into Myc stabilization in breast cancer involving aberrant Axin1 expression. Proc Natl Acad Sci. 2012 Feb 21;109(8):2790-5.

45. Huang X, Gao L, Wang S, McManaman JL, Thor AD, Yang X, et al. Heterotrimerization of the Growth Factor Receptors erbB2, erbB3, and Insulin-like Growth Factor-I Receptor in Breast Cancer Cells Resistant to Herceptin Cancer Res. 2010 Feb 1·70(3):1204-14

46. Sirsi SR, Hernandez SL, Zielinski L, Blomback H, Koubaa A, Synder M, et al. Polyplex-microbubble hybrids for ultrasound-guided plasmid DNA delivery to solid tumors. J Control Release Off J Control Release Soc. 2012 Jan 30;157(2):224-34.

47. Hayward WS, Neel BG, Astrin SM. Activation of a cellular onc gene by promoter insertion in ALV-induced lymphoid leukosis. Nature. 1981 Apr 9;290(5806):475-80.

48. Payne GS, Bishop JM, Varmus HE. Multiple arrangements of viral DNA and an activated host oncogene in bursal lymphomas. Nature. 1982 Jan 21;295(5846):209-14.

49. Alitalo K, Schwab M, Lin CC, Varmus HE, Bishop JM. Homogeneously staining chromosomal regions contain amplified copies of an abundantly expressed cellular oncogene (c-myc) in malignant neuroendocrine cells from a human colon carcinoma. Proc Natl Acad Sci. 1983 Mar 1;80(6):1707-11.

50. Brodeur G, Seeger R, Schwab M, Varmus H, Bishop J. Amplification of N-myc in untreated human neuroblastomas correlates with advanced disease stage. Science. 1984 Jun 8;224(4653):1121-4.

51. Hemann MT, Bric A, Teruya-Feldstein J, Herbst A, Nilsson JA, Cordon-Cardo C, et al. Evasion of the p53 tumour surveillance network by tumour-derived MYC mutants. Nature. 2005 Aug 11;436:807.

52. Dang CV. MYC on the Path to Cancer. Cell. 2012 Mar;149(1):22-35. 
53. Matkar S, Sharma P, Gao S, Gurung B, Katona BW, Liao J, et al. An Epigenetic Pathway Regulates Sensitivity of Breast Cancer Cells to HER2 Inhibition via FOXO/c-Myc Axis. Cancer Cell. 2015 Oct;28(4):472-85.

54. Borowicz S, Van Scoyk M, Avasarala S, Karuppusamy Rathinam MK, Tauler J, Bikkavilli RK, et al. The Soft Agar Colony Formation Assay. J Vis Exp [Internet]. 2014 Oct 27 [cited 2018 Aug 2];(92). Available from: http://www.jove.com/video/51998/the-soft-agar-colony-formation-assay

55. Horibata S, Vo TV, Subramanian V, Thompson PR, Coonrod SA. Utilization of the Soft Agar Colony Formation Assay to Identify Inhibitors of Tumorigenicity in Breast Cancer Cells. J Vis Exp [Internet]. 2015 May 20 [cited 2018 Aug 2];(99). Available from: http://www.jove.com/video/52727/utilizationsoft-agar-colony-formation-assay-to-identify-inhibitors

56. Liu E, Thor A, He M, Barcos M, Ljung BM, Benz C. The HER2 (c-erbB-2) oncogene is frequently amplified in in situ carcinomas of the breast. Oncogene. 1992 May;7(5):1027-32

57. Chang C-H, Scott GK, Kuo W-L, Xiong X, Suzdaltseva Y, Park JW, et al. ESX: a structurally unique Ets overexpressed early during human breast tumorigenesis. Oncogene. 1997 Apr;14(13):1617-22

58. Neve RM, Ylstra B, Chang C-H, Albertson DG, Benz CC. ErbB2 Activation of ESX gene expression. Oncogene. 2002 May;21(24):3934-8.

59. Eckel KL, Tentler JJ, Cappetta GJ, Diamond SE, Gutierrez-Hartmann A. The Epithelial-Specific ETS Transcription Factor ESX/ESE-1/Elf-3 Modulates Breast Cancer-Associated Gene Expression. DNA Cell Biol. 2003 Feb;22(2):79-94.

60. Zhou Y, Kumon RE, Cui J, Deng CX. The size of sonoporation pores on the cell membrane. Ultrasound Med Biol. 2009;35(10):1756-60.

61. Fan Z, Liu H, Mayer M, Deng CX. Spatiotemporally controlled single cell sonoporation. Proc Natl Acad Sci. 2012;109(41):16486-16491.

62. Lentacker I, Wang N, Vandenbroucke RE, Demeester J, De Smedt SC, Sanders NN. Ultrasound exposure of lipoplex loaded microbubbles facilitates direct cytoplasmic entry of the lipoplexes. Mol Pharm. 2009 Apr;6(2):457-67.

63. Yan $\mathrm{F}$, Li X, Jin $\mathrm{Q}$, Zhang Z, Lu M, Zheng $\mathrm{H}$. Therapeutic ultrasound microbubbles carrying paclitaxel and LyP-1 peptide: Preparation, characterization and application to ultrasonic assisted chemotherapy in breast cancer cells. In IEEE; 2010 [cited 2018 Aug 2]. p. 1571-4. Available from: http://ieeexplore.ieee.org/document/5935604/

64. Liu H-L, Hua M-Y, Chen P-Y, Chu P-C, Pan C-H, Yang H-W, et al. Blood-brain barrier disruption with focused ultrasound enhances delivery of chemotherapeutic drugs for glioblastoma treatment. Radiology. 2010 May;255(2):415-25.

65. Chen $\mathrm{H}$, Hwang J. Ultrasound-targeted microbubble destruction for chemotherapeutic drug delivery to solid tumors. J Ther Ultrasound. 2013;1(1):10.

66. Kotopoulis S, Dimcevski G, Mc Cormack E, Postema M, Gjertsen BT, Gilja OH. Ultrasound- and microbubble-enhanced chemotherapy for treating pancreatic cancer: A phase I clinical trial. J Acoust Soc Am. 2016 Apr;139(4):2092-2092.

67. Carpentier A, Canney M, Vignot A, Horodyckid C, Goldwirt L, Leclercq D, et al. Temporary disruption of the blood-brain barrier using an implantable ultrasound system for recurrent glioblastoma patients under IV carboplatin chemotherapy: initial phase 1/2a clinical trial observations. J Ther Ultrasound. 2015;3(Suppl 1):O14.

68. Khokhlova TD, Haider $Y$, Hwang JH. Therapeutic potential of ultrasound microbubbles in gastrointestinal oncology: recent advances and future prospects. Ther Adv Gastroenterol. 2015 Nov;8(6):384-94.

69. Hassan M, Barrefelt A, Saghafian M, Kuiper $R$, Ye, Egri $G$, et al. Biodistribution, kinetics, and biological fate of SPION microbubbles in the rat. Int J Nanomedicine. 2013 Aug;3241.

70. Fix SM, Nyankima AG, McSweeney MD, Tsuruta JK, Lai SK, Dayton PA. Accelerated Clearance of Ultrasound Contrast Agents Containing Polyethylene Glycol is Associated with the Generation of Anti-Polyethylene Glycol Antibodies. Ultrasound Med Biol. 2018 Jun;44(6):1266-80.

71. Hauff P, Seemann S, Reszka R, Schultze-Mosgau M, Reinhardt M, Buzasi T, et al. Evaluation of Gas-filled Microparticles and Sonoporation as Gene Delivery System: Feasibility Study in Rodent Tumor Models. Radiology. 2005 Aug;236(2):572-8.

72. Sakakima Y, Hayashi S, Yagi Y, Hayakawa A, Tachibana K, Nakao A. Gene therapy for hepatocellular carcinoma using sonoporation enhanced by contrast agents. Cancer Gene Ther. 2005 Nov;12(11):884-9.

73. Zhou S, Li S, Liu Z, Tang Y, Wang Z, Gong J, et al. Ultrasound-targeted microbubble destruction mediated herpes simplex virus-thymidine kinase gene treats hepatoma in mice. J Exp Clin Cancer Res. 2010;29(1):170.

74. McDannold N, Arvanitis CD, Vykhodtseva N, Livingstone MS. Temporary Disruption of the Blood-Brain Barrier by Use of Ultrasound and Microbubbles: Safety and Efficacy Evaluation in Rhesus Macaques. Cancer Res. 2012 Jul 15;72(14):3652-63

75. Miller D, Smith N, Bailey M, Czarnota G, Hynynen K, Makin I. Overview of Therapeutic Ultrasound Applications and Safety Considerations. J Ultrasound Med Off J Am Inst Ultrasound Med. 2012 Apr;31(4):623-34.

76. Downs ME, Buch A, Sierra C, Karakatsani ME, Chen S, Konofagou EE, et al. Long-term safety of repeated blood-brain barrier opening via focused ultrasound with microbubbles in non-human primates performing a cognitive task. PloS One. 2015;10(5):e0125911. 